\title{
Clinical and Anthropometric Characteristics of the Metabolically Unhealthy Phenotype in Adolescents with Adequate and High BMI
}

\section{Ana Carla Leocadio Magalhães ( $\sim$ acarlaleocadio@gmail.com )}

Universidade Federal do Rio de Janeiro https://orcid.org/0000-0002-9050-9980

Anna Paola T. Pierucci

Universidade Federal do Rio de Janeiro

Maria Núbia G. Oliveira

Universidade Federal do Rio de Janeiro

Aline B. F. Campos

Universidade Federal do Rio de Janeiro

\section{Patrícia C. Jesus}

Universidade Federal do Rio de Janeiro

Andrea Ramalho

Universidade Federal do Rio de Janeiro

Research article

Keywords: Menarche, Leptin, Metabolically health obesity, Body mass index, Adolescents

Posted Date: March 17th, 2020

DOl: https://doi.org/10.21203/rs.3.rs-17260/v1

License: (9) (i) This work is licensed under a Creative Commons Attribution 4.0 International License.

Read Full License 


\section{Abstract}

Background The metabolically healthy $(\mathrm{MH})$ and metabolically unhealthy $(\mathrm{MUH})$ phenotypes have been discussed in the literature but the factors related to the development of these phenotypes, in adolescents with adwuate and high body mass index (BMI), are not yet fully understood. Objective To evaluate the relationship between age during the menarche, serum leptin concentrations, body composition and $\mathrm{MH}$ and MUH phenotypes, in adolescents with adequate and high BMI.

Methods Observational and cross-sectional study made up of female adolescents attended at Centro de Referência do Adolescente, in Macaé, Rio de Janeiro. The stage of sexual maturation and the age at menarche were analyzed based on the self-declaration of the participants. The menarche was considered early (EM) when the first menstruation occurred up to 11 years of age; normal (NM) between 12 and 14 years old; and late after 15 years of age. Body mass and height were measured to calculate BMI, and the following body variables were analyzed: waist circumference (WC), waist/height ratio (WHR), body fat (BF\%), fat body mass index (FBMI) and fat-free mass index (FFMI), visceral adiposity index (VAI). In addition, cardiometabolic components and serum leptin concentrations were measured.

Results $82 \%(n=114)$ of the adolescents were classified as $\mathrm{MH}$ and $18 \%(n=25)$ as MU. The mean age at menarche was $11.79 \pm 1.39$ years. $60 \%(n=15)$ of $E M$ and $84 \%(n=21)$ of inadequacy of serum leptin were identified in the MUH group. Among eutrophic women, there was $85.7 \%(n=6)$ of inadequate WC among MUH adolescents and $2.5 \%(n=2)$ among $\mathrm{MH}$ ones $(\mathrm{p}<0.01)$, while among adolescents with high $\mathrm{BMI}$, $88.9 \%(n=16)$ and $28.6(n=10)$ of the MUH and MH groups, respectively, showed inadequacy of this variable $(p<0.01)$. Among MUH adolescents, there was a strong correlation between the age at menarche and BF\% $(r=-0.63 p<0.01)$; VAl and TG $(r=0.87 p<0.01), B F \%(r=0.63 p<0.01)$ and FFM $(r=0.61 p<0.01)$.

Conclusion Age at menarche, serum leptin and WC are related to the MUH phenotype in adolescents and should be investigated, during clinical practice, to help prevent cardiometabolic diseases in adulthood.

\section{Background}

The expressive growth of the prevalence of overweight and obesity in children and adolescents is considered a big problem of the world public health, because changes in blood pressure, blood glucose and lipid profile, that follow the excess of body mass, may contribute for the development of cardiometabolic diseases during adulthood [1, 2].

Recently, however, individuals have been identified who present obesity, according with the body mass index (BMI), and a less compromised metabolic profile, denominated metabolically healthy (MH), whose prevalence varies between $6 \%$ and $36 \%$ among the population of children and adolescents [3]. In contrast, it is estimated that nearly $20 \%$ of the subjects considered eutrophic by the BMI are classified as metabolically unhealthy $(\mathrm{MUH})$, due to presenting alterations in various cardiometabolic components [4, $5]$. 
Although the factors related to the development of these phenotypes are not yet fully understood, it is believed that individuals with $\mathrm{MH}$ obesity present differentiated body composition, especially regarding the distribution of body fat, better lipidic profile and greater adequacy of hormones secreted by the adipose tissue, as the leptin, when compared to the MUH eutrophics.

Body composition and serum leptin, besides relating to the $\mathrm{MH}$ and $\mathrm{MUH}$ phenotypes, seem to be determinants to the pubertal development, especially among female adolescents, because hormonal and bodily changes, which occur during the sexual maturation phase, are fundamental for the appearance of the first menstruation $[7,8]$.

In the last decades, concomitantly with the increase of prevalence of overweight and obesity, and, consequently, with the elevated inadequacy of serum leptin concentrations in young populations, a significant reduction in the age at menarche has been observed, which has worried scientific communities, since evidence shows that early menarche is also a risk factor for the development of cardiometabolic diseases in adulthood $[9,10]$. Despite that, to the best of our knowledge, there are no evidences linking the age at menarche with metabolic phenotypes. Thus, the aim of the present study is to assess the relationship between age at menarche, serum leptin concentrations, body composition and $\mathrm{MH}$ and $\mathrm{MUH}$ phenotypes in adolescents with adequate and elevated BMI.

\section{Materials And Methods Study Population}

This cross-sectional study investigated female adolescents who were patients at a reference health center for adolescents (Centro de Referência do Adolescente) in Macaé, Rio de Janeiro state, Brazil. Posters about the study were displayed in the waiting room, and the overall adolescents who were patients at the center from February 2013 to July 2013 were invited to take part in the study.

Girls aged 10 to 19 who had medical records at the health clinic and had gone through menarche were included. Exclusion criteria were to be pregnant or lactating, to have physical conditions that prevented their anthropometric variables from being measured adequately and use of antipsychotic drugs.

The study was approved by the Research Ethics Committee of Hospital Universitário Clementino Fraga Filho, the teaching hospital attached to the Federal University of Rio de Janeiro. Informed consent forms were signed by the overall participants - by the adolescents who were aged 18 or over and by the parents/guardians of those who were under 18.

\section{Sexual Maturity and Age at Menarche}

Sexual maturity was self-assessed using a form showing the stages of breast development, based on the Tanner stages. Stage 1 was classified as prepubescent, while stages II, III, and IV were classified as pubescent, and stage $V$ was classified as postpubescent $[11,12]$. 
Information on menarche was obtained by asking the subjects their age (in years and months) and the time of their first period. Early menarche was $\leq 11$ years old, normal menarche was $12-15$ years old, and late menarche was $\geq 15$ years old [13].

\section{Anthropometric Variables}

Weight and height were measured using the techniques recommended by Jellife [14], with the subjects barefoot and wearing shorts and a top. They were weighed on digital scales (Welmy, Santa Bárbara d'Oeste, Brazil) and their height was measured using a stadiometer (model 120; Tonelli, Criciúma, Brazil). Body mass index $(\mathrm{BMI})$ was calculated by dividing body weight $(\mathrm{kg})$ by height $\left(\mathrm{m}^{2}\right)$. Nutritional status was classified using the cutoff points established by the WHO [15] and the Brazilian nutrition surveillance system, Sistema de Vigilância Alimentar [16], in which normal weight is $\geq 3$ rd percentile and $<85$ th percentile, overweight is $\geq 85$ th percentile, obesity is $\geq 97$ th percentile, and severe obesity is $\geq 99$ th percentile.

In order to assess body weight distribution and cardiovascular risk, the girls' waist circumference (WC) was measured using a flexible tape placed midway between the lowest rib and the iliac crest, as recommended by McCarthy [17]. The cutoff point used for calculating the inadequacy of WC was the 90th percentile, according to Fernandéz et al. [18].

The waist-to-height ratio (WHR) was calculated dividing WC $(\mathrm{cm})$ by height $(\mathrm{cm})$ and the cutoff point value adopted for this variable was 0.50 [19]

To estimate the amount of visceral adipose tissue, the visceral adiposity index (VAl) was calculated using the variables WC $(\mathrm{cm}), \mathrm{BMI}\left(\mathrm{kg} / \mathrm{m}^{2}\right), \mathrm{TG}(\mathrm{mmol} / \mathrm{L})$ and $\mathrm{HDL}-\mathrm{c}(\mathrm{mmol} / \mathrm{L})$, according to a formula proposed by Amato et al. [20]:

VAI $_{\text {women }}=\left(\frac{W C}{36.58+(1.89 x B M I)}\right) \times\left(\frac{T G}{0.81}\right) \times\left(\frac{1.52}{H D L-c}\right)$

Body fat percentage (BF\%) was calculated using the equations of Slaugther et al. [21]. The cutoff point value adopted for elevating $B F \%$ was $\geq 30 \%$, as recommended by Willians et al. [22]. From this variable, fat body mass (FBM) was estimated in pounds, and lean body mass (LBM) was calculated by subtraction between total body mass $(\mathrm{kg})$ and FBM $(\mathrm{kg})$ [23]. Then, the fat body mass index (FBMI) and the fat-free mass index (FFMI) were calculated, according to the equations proposed by Vanitallie et al. [24]:

FBMI $=\frac{F B M(\mathrm{~kg})}{\text { Height }\left(\mathrm{m}^{2}\right)}$

FFMI $=\frac{\operatorname{LBM}(\mathrm{kg})}{\text { Height }\left(\mathrm{m}^{2}\right)}$ 


\section{Blood Pressure and Blood Tests}

Blood pressure was measured by the oscillometric method using a calibrated semi-automatic digital device. Two measurements were taken one minute apart, and mean was calculated.

Blood was drawn at least after a 12-hour fast for laboratory tests. Blood was taken from the antecubital vein by a trained nurse and stored in plastic tubes with separator gel and clot activator. Tubes were centrifuged at 2,000 RCF (relative centrifugal force) to separate out the plasma and serum and then frozen at $-80^{\circ} \mathrm{C}$ until the analyses. The methods used to analyze triglycerides, HDL-C, and glucose were a lactate dehydrogenase UV assay, a direct method, and an enzymatic colorimetric method (using hexokinase), respectively. Serum concentrations of leptin were analyzed using an ELISA kit (CAN-L4260, DBC Inc., Canada) with the Basic Robotic Immunoassay Operator (BRIO, Italy). The cutoff point for adequate serum leptin was set at $>11.1 \mathrm{ng} / \mathrm{mL}$, as recommended in the kit [25].

\section{Metabolic Classification}

The adolescent girls were classified as metabolically healthy $(\mathrm{MH})$ and metabolically unhealthy $(\mathrm{MUH})$ using the criteria established by the National Cholesterol Education Program Adult Treatment Panel III (NCEP-ATP III) [26]. They were classified as MUH if at least three of the following criteria applied to them: WC $\geq 90$ th percentile [18]; triglycerides $\geq 150 \mathrm{mg} / \mathrm{dL} ; \mathrm{HDL}-\mathrm{C} \leq 50 \mathrm{mg} / \mathrm{dL}$; fasting blood glucose $\geq$ $100 \mathrm{mg} / \mathrm{dL}$ [27]; blood pressure $\geq 90$ th percentile [28].

\section{Statistical analysis}

For statistical analysis, the adolescents were stratified according to age groups, metabolic classification and BMI. The normality of the sample distribution was evaluated by the Kolmogorov-Smirnov test.

Descriptive analyses and the independent samples t-test were used to general characterization of the sample and the equality of means was analyzed by the Levene test.

The association between the categorical variables was analyzed by the Chi-square test $\left(x^{2}\right)$ and the association between the continuous variables by the Pearson correlation. In this study, strong correlation occurred when values were above 0.6 , moderate correlation when values were between 0.4 and 0.59 and low correlation when values were below 0.39 [29].

The level of statistical significance adopted was $p<0.05$. Analyses were carried out in the SPPS program, version 21.0.

\section{Results}

A total of 139 adolescents participated in the study, with a mean age at menarche of $11.79 \pm 1.39$ years and $\mathrm{BMI}$ of $23.52 \pm 5.41 \mathrm{~kg} / \mathrm{m}^{2}$. The MH and MUH phenotypes were prevalent in $82 \%(n=114)$ and $18 \%$ $(n=25)$ of the sample, respectively. This last one presented slightly higher averages of BMI, WC, WHR, FBMI and smaller averages of HDL-c, when compared with the MH group (Table 1). 
There was a higher prevalence of EM $(63.0 \%, n=29)$ and inadequacy of leptin $(80.0 \%, n=36)$ among adolescents who were aged between 10 and 14 years when compared to those older than $15(p<0.01)$. Regarding the metabolic phenotypes, $52.0 \%(n=13)$ of the MUH adolescents were in the pubertal phase of sexual maturation; $60.0 \%(n=15)$ presented EM $(p=0.04), 84.0 \%(n=21)$ demonstrated high serum leptin $(p=0.01)$. Regarding the BMI, $28.0 \%(n=7)$ of the MUH group was classified as eutrophic, while $30.7 \%(n=35)$, of the MH adolescents had a BMI $\geq p 85(p<0.01)$ (Table 2$)$.

Among the eutrophic ones, a higher prevalence of WC inadequacy was identified in the MUH group $(85.7 \%, n=6)$, when compared to the $\mathrm{MH}$ one $(2.5 \%, \mathrm{n}=2)(\mathrm{p}<0.01)$. With regard to adolescents with a $\mathrm{BMI} \geq \mathrm{p} 85$, there was $61.1 \%(\mathrm{n}=11)$ and $28.1 \%(\mathrm{n}=9)$ of HDL-c inadequacy in MUH and MH groups, respectively $(p=0.02) ; 88.9 \%(n=16)$ and $28.6 \%(n=10)$ of PC inadequacy in MUH and MH adolescents, respectively $(p<0.01)$; and $77.8 \%(n=14)$ in MUH ones and $48.6 \%(n=17)$ in MH ones with elevated WHR $(p=0.04)$ (Table 3$)$.

The correlation analysis between the variables, according to the $\mathrm{MH}$ and $\mathrm{MUH}$ phenotypes, is shown in Table 4. There was a strong correlation between $\mathrm{BMI}$ and other anthropometric variables, with emphasis on WC, WHR, FBMI and FFMI in both groups. Among the MUH adolescents, a moderate and negative correlation was identified between $\mathrm{BMI}$ and age at menarche $(r=-0.41 ; p<0.05)$ and a positive correlation between BMI and serum leptin $(r=0.53 ; p<0.01)$.

\section{Discussion}

The MH and MUH phenotypes have recently been discussed in the literature [30, 31]. However, to our knowledge, this is the first study that evaluates the association of these metabolic profiles with the age at menarche, serum leptin concentrations and body variables, in eutrophic adolescents and those with excess of body mass.

Our data demonstrate a higher frequency of MUH adolescents in the pubertal phase of sexual maturation. Similarly, Reinehr et al. [32], in a longitudinal study with obese children and adolescents, observed that pubescent children were twice as likely to have an inadequate metabolic profile than those who were in the pre- or post-pubertal phase of sexual maturation.

Considering that the menarche is the main marker of sexual maturation in females, some authors have identified an association between age at first menstruation and the development of cardiometabolic risk factors during adulthood [33,34]. However, with regard to metabolic phenotypes, studies that address this relationship have not yet been found.

In this present study, a high frequency of EM was identified among MUH adolescents with adequate and high BMI, and among those who were between 10 and 14 years old. These findings are relevant, since several authors have pointed to a trend of reduction in the age at menarche in recent decades, which can be justified by important changes in body composition that occur during puberty $[35,36,8]$. In our data, 
among MUH adolescents, the significant correlation found between the age at menarche and body variables, such as $\mathrm{BMI}$ and $\mathrm{BF} \%$, corroborate this hypothesis.

The amount and distribution of body fat, in addition to being important influencers of menarche age, interfere with serum leptin concentrations, since adipose tissue is the main organ responsible for the production of this hormone $[37,7,8]$. In this sense, we highlight the high inadequacy of serum leptin levels, found in our sample, especially among adolescents with high BMI and MUH phenotype, as observed by Ding et al [38]. Despite this, it is noteworthy that, in the present study, high serum concentrations of this adipokine were also found between eutrophic and $\mathrm{MH}$ adolescents. This fact can be justified by the higher prevalence of adolescents in the pubic phase of sexual maturation, since pubertal staging is an important factor related to leptinemia in eutrophic individuals [39].

WC and WHR are anthropometric variables frequently used to estimate the distribution of body fat and the risk of developing cardiometabolic complications, as they have a strong association with insulin resistance markers and inflammatory biomarkers, such as $\mathrm{C}$-reactive protein and adiponectin. However, there are still doubts about which of these variables is considered a better indicator of the MUH phenotype $[31,40,41,42,43]$.

The findings of the present study demonstrate that WC was a more adequate predictor for the MUH phenotype than WHR, since a high inadequacy of this variable was observed, with a statistically significant difference, when compared to the $\mathrm{MH}$ and $\mathrm{MUH}$ groups, in eutrophic adolescents and the ones with high BMI. In addition, WC presented a higher correlation with VAI, in the MUH group, than WHR.

VAl is an anthropometric evaluation method created to estimate the amount of visceral adipose tissue (VAT), since this compartment is strongly related to the development of insulin resistance, metabolic syndrome, diabetes mellitus and cardiovascular diseases [39, 40, 41]. Regarding the $\mathrm{MH}$ and $\mathrm{MUH}$ phenotypes, Hwang et al [44] demonstrated that the accumulation of VAT contributed to individuals, initially classified as MH by the NCEP-ATP III [26], becoming MUH over a ten-year period.

Although the accumulation of VAT is an important risk factor for several cardiometabolic diseases in adults, it is believed, however, that, in the pediatric phase, metabolic components such as insulin resistance and high serum concentrations of TG may be better predictors of unfavorable metabolic outcomes [45]. In this sense, it is emphasized that, in the present study, there was a strong correlation between VAI and TG in the MUH group. In addition, MUH adolescents had higher mean VAI when compared to the $\mathrm{MH}$ ones, although without statistical significance. These findings become relevant due to the strong association found between this index and the MH and MUH phenotypes, as demonstrated by Kang et al [46].

It is also noteworthy the strong correlation found between $\mathrm{VAI}, \mathrm{BF} \%$ and $\mathrm{FFBI}$, in the MUH group, and the highest means of these variables, observed in adolescents with inadequate phenotype. These data corroborate with results demonstrated by Oliveira et al. [47] who, when analyzing factors related to cardiometabolic risk in adolescents, identified that the overweight group had a higher FFBI when 
compared to subjects with adequate BMI and WC. This characteristic can be justified by behavioral changes, such as decreased level of physical activity and poor eating habits, in addition to changes in glucose and lipid metabolism, which are common during adolescence [48, 42].

Data found in our study demonstrate that EM and serum leptin inadequacy are factors related to the MUH phenotype and that WC is an important risk indicator for this metabolic profile in eutrophic adolescents and the ones with high BMI. These findings illustrate the importance of this work since, to date, there are no studies that investigate the connection between these variables and the $\mathrm{MH}$ and MUH phenotypes, especially in adolescents in different classes of BMI. However, this study has limitations such as the reduced sample, the cross-sectional model, which prevents the analysis of causality between the variables analyzed, and the non-evaluation of the use of contraceptive drugs that could interfere with the investigated laboratory parameters.

\section{Conclusion}

During clinical practice, with individuals in the pediatric phase, it is recommended to investigate age at menarche, serum concentrations of leptin and WC in eutrophic and overweight individuals, for possible identification of the MUH phenotype, so that strategies to prevent the development of cardiometabolic diseases in adulthood can be developed.

\section{Abbreviations}

$\mathrm{MH}$

Metabolically healthy; MUH-Metabolically unhealthy; EM-Early menarche; NM - Normal menarche; LMLate menarche; BMI-Body mass index; WHO-World Healthy Organization; WC-Waist circumference; WHR-Waist-to-height ratio; TG-Triglycerides; HDL-C-High density lipoprotein cholesterol; VAI-Visceral adiposity index; BF\% - Body fat percentage; FBM-Fat mass body; LBM-lean body mass; FBMI - Fat body mass index; FFMI - Fat-free mass index; BP-Blood pressure; SBP-Systolic blood pressure; DBP-Diastolic blood pressure; NCEP-ATP III - National Cholesterol Education Program Adult Treatment Panel III; VATVisceral adiposity tissue.

\section{Declarations}

Ethics approval and consent to participate

The study and method of consense were approved by the Research Ethics Committee at Hospital Universitário Clementino Fraga Filho, the teaching hospital attached to the Federal University of Rio de Janeiro. Informed consent forms were signed by all the participants - by the adolescents who were aged 18 or over and by the parents/guardians of those under 18 .

Consent for publication

Not applicable. 
Availability of data and materials

The datasets generated and/or analyzed during the current study are not publically available because of privacy concerns of the participants and because they are property of the Micronutrient Research Center/Institute of Nutrition Josué de Castro/Federal University of Rio de Janeiro, but are available from the corresponding author on reasonable request.

Competing of Interest

The authors declare that they have no conflicts of interest.

\section{Funding Statement}

This study was support by funding from Fundação Carlos Chagas Filho de Amparo à Pesquisa do Estado do Rio de Janeiro (FAPERJ - Carlos Chagas Filho Foundation for Research Support of the State of Rio de Janeiro; PENSA/RIO public notice - FAPERJ 199009285; Scientist of Our State), Conselho Nacional de Desenvolvimento Científico e Tecnológico (CNPq - National Council for Scientific and Technological Development) and CAPES - Brazilian Federal Agency for Support and Evaluation of Graduate Education within the Ministry of Education of Brazil.

Authors' contributions

ACLM research data, analyzed data and wrote the first version of the manuscript. APTP reviewed the analyses and the manuscript. NGO and ABFC collected the data. PCJ analyzed data and reviewed the manuscript. AR supervised collection of the data, reviewed the analyses and reviewed critically the manuscript. All authors read and approved the final manuscript.

Acknowledgments

Not applicable.

\section{References}

1.

WORLD HEALTH ORGANIZATION (WHO). Report of Commission on Ending Childhood Obesity. Geneva: World Health Organization; 2016.

2.

Faria SL, Faria OP, Menezes CS, Gouvêa HR, Cardeal MA. Metabolic profile of clinically severe obese patients. Obes Surgy. 2012;22(8):1257-62.

3.

Ortega FB, Cadenas-Sánchez C, Sui X, Blair SN, Lavie CJ. Role of Fitness in the metabolically healthy but obese phenotype: a review and update. Prog Cardiovasc Dis. 2015;58(1):76-86.

4.

Lopez-Garcia E, Guallar-Castillón P, Garcia-Esquinas E, Rodríguez-Artalejo F. Metabolically health obesity and health - related quality of life: a prospective cohort study. Clin Nutr. 2017;36(3):36: 853-60.

5 .

Mathew H, Farr OM, Mantzoros CS. Metabolic health and weight: understanding unhealthy normal weight or metabolically healthy obese patients. Metabolism. 2016;65(1):73-80. 
6.

Karelis AD, St-pierr DH, Conus, Rabasa-lhoret R, PoehIman ET. Metabolic and body composition factors in subgroups of obesity: what do we know? J Clin Endocrinol Metab. 2004;89(6):2569-75.

7.

Von Schnurbein J, Moss A, Nagel SA, Muehleder H, Debatin KM. Farooqi IS.

et al. Leptin substitution results in the induction of menstrual cycles in adolescent with leptin d eficiency and hypogonadotropic hypogonadism. Horm Res Paediatr.2012;77:127-33.

8.

Shalitin S, Kiess W. Putative effects of obesity on linear growth and puberty. Horm Res Paediatr. 2017;8(1):101-10.

9.

Mishra HD, Chunga HF, Pandeyaa N, Dobsona AJ, Jonesa L, Avisb NE, et al. The interlace study: design, data harmonization and characteristics across 20 studies on women's health. Maturitas. 2016;92:17685.

10.

Day FR, Elks CE, Murray A, Ong KK, Perry JRB. Puberty timing associated with diabetes, cardiovascular disease and also diverse health outcomes in men and women: the UK Biobank study. Sci Rep. 2015;18(5). 11.

Tanner JM. Growth in adolescence. 2 ed. Oxford: Blackwell Scientific Publication; 1962.

12.

Chipkevitch E. Clinical evaluation of sexual maturity in adolescence. J Pediatr (Rio J). 2001;77(supl.2):S135-42.

13.

Stöckl D, Meisinger C, Peters A, Thorand B, Huth C, Heier M, et al. Age at menarche and its association with the metabolic syndrome and its components: results from the kpra f4 study. PLoS One. 2011:6(10). 14.

Jellife DB.Evaluation of the nutritional status of the community with special reference to the costs in the developing regions. In: World health Organization, ed. Genebra1968.

15.

WORLD HEALTH ORGANIZATION (WHO).Growth Reference Data 5-19 years. BMI-for-age (5-19 years).Percentiles, 2007.

16.

Ministry of Health of Brazil. Protocols of Feeding and Nutritional Surveillance. System. - SISVAN in health care (Brazil), 2008.

17.

McCarthy HD, Jarrett KV, Crawley HF. The development of waist circumference percentiles in British children ages 5.0-16,9 y. Eur J Clin Nutr. 2011;55(10):902-7.

18.

Fernandéz JR, Redden DT, Pietrobelli A, Allison DB. Waist circumference percentiles in nationally representative samples of African-American, European-American, and Mexican-American children and 
adolescents. J Pediatr. 2004;145(4):439-44.

19.

McCarthy HD, Ashwell M. A study of central fatness using waist-to-height ratios in UK children and adolescents over two decades supports the simple message - 'keep your waist circumference to less than half your height'. Int J Obes (Lond). 2006;30(6):988-92.

20.

Amato MC, Giordano C, Galia M, Criscimanna A, Vitabille S, Midiri M, et al. Visceral adiposity index: a reliable indicator of visceral fat function associated with cardiometabolic risk. Diabetes Care. 2010;33:920-2.

21.

Slaugther MH, Lohmann TG, Boileau AR, Horswill CA, Stillman RJ Van. Loan.

MD. et al. Skinfold equations for estimation of body fatness in children and youth. Hum Biol. 1988;60:709-23.

22.

Williams DP, Going SB, Lohman TG, Harsha DW, Snnivasan SR, Webber LS.

et al. Body fatness and risk for elevated blood pressure, total cholesterol and serum lipoprotein ratios in children and adolescents. Am J Public Health.

1992.;82(3):358-63.

23.

Ronque ERV, Guariglia DA, Cyrino ES, Carvalho FO, Avelar A, Arruda M. Composição corporal em crianças de sete a 10 anos de idade, de alto nível socioeconômico. Rev Bras de Med Esporte. 2007;13(6):366-70. 24.

Vanitallie TB, Yang UM, Heymsfield SB, Funk RC. RA B. Height-normalized indices of the body's fat-free mass and fatmass: potentially useful indicators of nutritional status. Am J Clin Nutr. 1990;52(8):953-9. 25.

Barbosa VS, Francescantônio PL, Silva NA. Leptin and adiponectin in patients with systemic lupus erythematosus: clinical and laboratory correlations. Rev Bras Reumatol. 2015;55(2):140-5. 26.

NCEP/ATPIII. Executive summary of the third report of the national cholesterol education program (NCEP) expert panel on detection, evaluation and treatment of high blood cholesterol in adults. JAMA. 2001;285(19):2486-97.

27.

Zimmet P, Alberti KG, Kaufman F, Tajima M, Silink M, Arslanian S, et al. The metabolic syndrome in children and adolescents - an IDF consensus report. Pediatric Diabetes. 2007;8:299-306. 28.

Brazilian Society of Cardiology; Brazilian Society of Hypertension. Brazilian Society of Nephrology. VI Brazilian Guideline on Hypertension. Arq Bras Cardiol. 2010;95(1 supl.1):1-51. 29.

Araujo AJS, Santos ACO, Prado WL. Body composition of obese adolescents: association between adiposity indicators and cardiometabolic risk factors. J Hum Nutr Diet. 2017;30(2):193-202. 
30.

Bervoets L, Massa G. Classification and clinical characterization of metabolically "health" obese children and adolescents. J Pediatr Endocrinol Metab. 2016;29(5):553-60.

31.

Lind L, Ärnlov J, Lampa E. The interplay between fat mass and fat distribution as determinants of metabolic syndrome is sex-dependent. Metab Syndr Relat Disord. 2017;15(7):337-43.

32.

Reinehr T, Wolters B, Knop C, Lass N, Holl RW. Strong effect of pubertal status on metabolic health in obese children: A longitudinal study. J Clin Endocrinol Metab. 2015;100(1):301-8.

33.

Mueller NT, Duncan BB, Barreto SM, Chor D, Bessel M, Estela ML, et al. Earlier age at menarche is associated with higher diabetes risk and cardiometabolic disease risk factors in brazilian adults: brazilian longitudinal study of adult health (ELSA - Brasil). Cardiovasc Diabetol. 2014;13:22.

34.

Sobrero AF, Evangelista P, Kovalskys I, Digón P, López S, Scaiola E, et al. Cardio-metabolic risk factors in Argentine children: a comparative study. Diabetes Metab Syndr. 2016;10(1):103 - S9.

35.

Won JC, Hong JW, Noh JH, Kim. D-J. Association between age at menarche and.

risk factors for cardiovascular diseases in Korean Women. Medicine (Beltimore). 2016;95(18). 36.

Elsehely I, Hafez HA, Ghonem M, Fathi A, Elzehery R. A cutoff for age at menarche predicting metabolic syndrome in Egyptian overweight/obese premenopausal women. Diabetes Metab J. 2017;41(2):146-9. 37.

Soliman AT, Yasin M, Kassem A. Leptin in pediatrics: A hormone from adipocyte that wheels several functions in children.. Indian J Endocrinol Metab. 2012;16(3):p.S577 - S87.. 38.

Ding W, Yan Y, Zhang M, Cheng H, Zhao X, Hou D, et al. Hypertension outcomes in metabolically unhealthy normal-weight and metabolically healthy obese children and adolescents. J Hum Hypertens. 2015;29(9):p. $548-54$.

39.

Almeida CAN, Ramos APP, Brunetti IL, Pepato MT, Ricco RG. Fasting leptinemia in eutrophic children and adolescents. Rev Assoc Med Bras. 2009;55(4):463-7.

40.

Heinzle S, Bal GDC, Kuk JL. Variations in the prevalence and predictors of prevalent metabolically healthy obesity in adolescents. Pediatr Obes. 2016;11(5):425-33.

41.

Reinehr T, Wolters B, Knop C, Lass N, Holl RW. Strong effect of pubertal status on metabolic health in obese children: A longitudinal study. J Clin Endocrinol Metab. 2015;100(1):301-8.

42. 
Oliveira RP, Remor JM, Matsuo AR, Dada RP, Mendes AA, Santos TL, et al. Visceral adiposity index as a predictor of cardiometabolic risk in children and adolescents. Rev Bras Med Esporte. 2017;23(3):222-6. 43.

Xia L, Dong F, Gong H, Xu G, Wang K, Liu F, et al. Association between indices of body composition and abnormal metabolic phenotype in normalweight chinese adults.. Int J Environ Res Public Health. 2017;14(391).

44.

Hwang YC, Hayashi T, Fujimoto WY, Kahn SE, Leonetti DL, McNeely MJ, et al. Visceral abdominal fat accumulation predicts the conversion of metabolically healthy obese subjects to an unhealthy phenotype. Int J Obes (Lond). 2015;39(9):1365-70.

45.

Ali O, Cerjak D, Kent JD Jr, James R, Blangero J, Zhang Y. Obesity, central adiposity and cardiometabolic risk factors in children and adolescents: a family-based study. Pediatr Obes. 2014;9(3):e58- e62.

46.

Kang YM, Jung $\mathrm{CH}$, Cho YK, Jang JE, Hwang JY, Kim EH, et al. Visceral adiposity index predicts the conversion of metabolically healthy obesity to an unhealthy phenotype. PIOs One. 2017;12(6):e0179635. 47.

Oliveira PM, Silva FA, Oliveira RMS, Mendes LL, Netto MP, Cândido APC. Association fat mass index and fat-free mass index values and cardiovascular risk in adolescents. Rev Paul Pediatr. 2016;34(1):30-7. 48 .

Weber DR, Leonard MB, Shults J, Zemel BS. A Comparison of fat and lean body mass index to BMI for the identification of metabolic syndrome in children and adolescents. J Clin Endocrinol Metab.

2014;99(9):3208-16. 